\title{
Chinese expert recommendation on transnasal corticosteroid nebulization for the treatment of chronic rhinosinusitis 2021
}

\author{
Chengshuo Wang ${ }^{1,2,3 \#}$, Lei Cheng ${ }^{4,5 \#}$, Huabin Li $^{6 \#}$, Zheng Liu ${ }^{7 \#}$, Hongfei Lou ${ }^{1,2,3 \#}$, Jianbo Shi ${ }^{8 *}$, \\ Ying Sun ${ }^{1,2,9 \#}$, Dehui Wang ${ }^{6 \#}$, Qintai Yang ${ }^{10 \#}$, Hongmeng Yu, ${ }^{6,11 \#}$, Changqing Zhao ${ }^{12 \#}$, Dongdong Zhu ${ }^{13 \#}$, \\ Fengli Cheng ${ }^{12}$, Yan $\mathrm{Li}^{1,2,3}$, Bo Liao ${ }^{7}$, Meiping Lu ${ }^{4}$, Cuida Meng ${ }^{13}$, Shen Shen ${ }^{1,2,3}$, Yueqi Sun ${ }^{14}$, Rui Zheng ${ }^{10}$, \\ Luo Zhang ${ }^{1,2,3,15}$; on behalf of the Group of Otorhinolaryngology and Ophthalmology, Chinese Society \\ of Allergy (CSA)
}

${ }^{1}$ Department of Otolaryngology Head and Neck Surgery, Beijing Tongren Hospital, Capital Medical University, Beijing, China; ${ }^{2}$ Beijing Laboratory of Allergic Diseases and Beijing Key Laboratory of Nasal Diseases, Beijing Institute of Otolaryngology, Beijing, China; ${ }^{3}$ Research Unit of Diagnosis and Treatment of Chronic Nasal Diseases, Chinese Academy of Medical Sciences, Beijing, China; ${ }^{4}$ Department of Otorhinolaryngology \& Clinical Allergy Center, the First Affiliated Hospital, Nanjing Medical University, Nanjing, China; ${ }^{5}$ International Centre for Allergy Research, Nanjing Medical University, Nanjing, China; ' Department of Otolaryngology Head Neck Surgery, Eye \& ENT Hospital of Fudan University, Shanghai, China; ${ }^{7}$ Department of Otolaryngology-Head and Neck Surgery, Tongji Hospital, Tongji Medical College, Huazhong University of Science and Technology, Wuhan, China; ${ }^{8}$ Otorhinolaryngology Hospital, the First Affiliated Hospital, Sun Yat-sen University, Guangzhou, China; ${ }^{9}$ Department of Immunology, School of Basic Medical Sciences, Capital Medical University, Beijing, China; ${ }^{10}$ Department of Otorhinolaryngology-Head and Neck Surgery, Department of Allergy, Third Affiliated Hospital, Sun Yat-sen University, Guangzhou, China; ${ }^{11}$ Research Units of New Technologies of Endoscopic Surgery in Skull Base Tumor, Chinese Academy of Medical Sciences, Shanghai, China; ${ }^{12}$ Department of Otorhinolaryngology-Head and Neck Surgery, Second Hospital of Shanxi Medical University, Taiyuan, China; ${ }^{13}$ Department of Otorhinolaryngology Head and Neck Surgery, China-Japan Union Hospital of Jilin University, Changchun, China; ${ }^{14}$ Department of Otolaryngology, the Seventh Affiliated Hospital, Sun Yat-sen University, Shenzhen, China; ${ }^{15}$ Department of Allergy, Beijing Tongren Hospital, Capital Medical University, Beijing, China

"These authors contributed equally to this work.

Correspondence to: Luo Zhang, MD, PhD. Beijing Tongren Hospital, No. 1, Dongiiaominxiang, Dongcheng District, Beijing 100005 , China.

Email: dr.luozhang@139.com.

\begin{abstract}
Corticosteroids are efficacious in treating chronic rhinosinusitis (CRS), but concerns on the potential side effects remain, especially for long-term usage of systemic corticosteroids. Accumulated evidence shows that transnasal nebulization may be a reasonable solution in balancing both efficacy and safety. However, no consensus or guideline has been formulated on the use of steroid transnasal nebulization in treating CRS. The consensus is achieved through literature review and exchange of Chinese experts in Group of Otorhinolaryngology and Ophthalmology, Chinese Society of Allergy (CSA). This document covers the development, equipment, pharmacological mechanism, and evidence-based efficacy and safety, as well as the special concern of the application of steroid transnasal nebulization during the coronavirus disease (COVID-19) pandemic. The expert consensus clarifies the application of steroid transnasal nebulization in treating CRS and common comorbidities during the perioperative and postoperative periods.
\end{abstract}

Keywords: Chronic rhinosinusitis (CRS); corticosteroids; efficacy; safety; transnasal nebulization

Submitted Jul 10, 2021. Accepted for publication Nov 16, 2021.

doi: $10.21037 /$ jtd-21-1142

View this article at: https://dx.doi.org/10.21037/jtd-21-1142

^ ORCID: 0000-0002-0910-9884.

(C) Journal of Thoracic Disease. All rights reserved. 


\section{Introduction}

Chronic rhinosinusitis (CRS), widely accepted as a highly heterogeneous inflammatory disease, affects $5.5-28 \%$ of the global population and exerts huge economic and social burdens (1). In China, the prevalence of CRS is approximately $8 \%$ (2). Frequent symptoms, such as nasal congestion, smell impairment, headache, and nasal discharge, substantially affect the quality of life of patients with CRS, often leading to sleep disturbance (3). Moreover, approximately a quarter of the patients have psychological dysfunction, which is associated with higher medication costs and a worse prognosis (4). Clinically, CRS is classified into two phenotypes: CRS with nasal polyps (CRSwNP) and CRS without nasal polyps (CRSsNP). The former phenotype is often associated with higher postoperative recurrence rate and severe clinical manifestations (5).

In the past, nasal sprays and oral corticosteroids were widely applied in daily clinical practice. Currently, corticosteroids, including nasal and systemic corticosteroids, have been listed as 1a level of evidence recommended therapy of European Position Paper on Rhinosinusitis and Nasal Polyps 2020 for treating adults with CRS, underscoring their efficacy and safety in treating CRS $(1,6)$. Although intranasal corticosteroid therapy can relieve nasal obstruction, rhinorrhea, and hyposmia and reduce the size of the polyps in CRSwNP patients, its effect of full recovery is limited (1,7). A short course of systemic corticosteroids could significantly improve the symptom scores and quality of life, especially in patients with partially or uncontrolled CRS (1). However, there are concerns about the potential side effects of long-term use of systemic corticosteroids, such as osteoporosis, suppression of hypothalamic-pituitaryadrenal axis (HPA), and obesity.

Transnasal nebulization is considered a reasonable and superior alternative to nasal sprays and systemic corticosteroids in balancing efficacy and safety. Several studies report that nebulization of sodium hyaluronate leads to better improvement in symptoms and quality of life than saline solution (8-11). Further, the efficacy and safety of nebulized corticosteroids in treating CRS have been proven by two well-designed clinical trials $(7,12)$. A rigorous head-to-head study indicated that a 2 -week transnasal nebulization of budesonide was not only more efficacious than nasal sprays but also safer than oral methylprednisolone (7).

Therefore, this expert consensus by a panel of Chinese rhinologists and immunologists was established to provide up-to-date information on the application of steroid transnasal nebulization in treating CRS.

We present the following article in accordance with the RIGHT reporting checklist (available at https://dx.doi. org/10.21037/jtd-21-1142).

\section{Transnasal nebulization}

Inhaled therapy has been the primary treatment for lung conditions for thousands of years (13). In recent decades, the focus has shifted to the role of transnasal nebulization in nasal diseases. The European Position Paper on Rhinosinusitis and Nasal Polyps 2020 shows that there is high-quality evidence that long-term use of nasal corticosteroids can effectively treat patients with CRS (1). Nasal corticosteroids can be administered through several methods, such as nasal spray, nasal drops, nasal irrigation, transnasal nebulization, and corticosteroid-eluting implants. Nebulizers have been used to treat respiratory diseases for many years, and the two basic types of nebulizers are jet and ultrasonic nebulizers (14). Several randomized controlled trials have confirmed that short-term steroid transnasal nebulization is an effective and safe treatment option for patients with CRS (7,15-17).

\section{Nebulization device}

The nebulization device comprises a mask or soft nose plug, nebulizer cup, and tubing.

\section{Environment and equipment}

\section{Environment}

The environment should be quiet, tidy, and safe. The nebulization device needs to be equipped with an oxygen flowmeter and hospital wall oxygen supply device. Besides, care is required for the nasal environment to ensure appropriate breathing and comfortable posture. In addition, healthcare providers should wash their hands and face after treatment. They should also clean the atomizer cup with clean water after each use and then atomize it with clear water for one minute to remove any residual liquid in the nebulizer cup.

\section{Equipment}

Oxygen-driven nebulization in the hospital needs to be equipped with an oxygen flowmeter (Figure 1) and a hospital wall oxygen supply device. The assembly and operation 


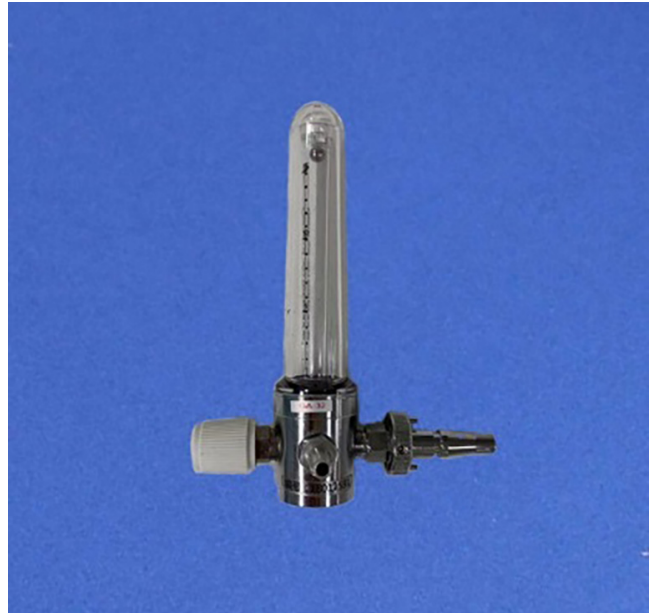

Figure 1 An oxygen flowmeter can be used to adjust the oxygen flow rate.

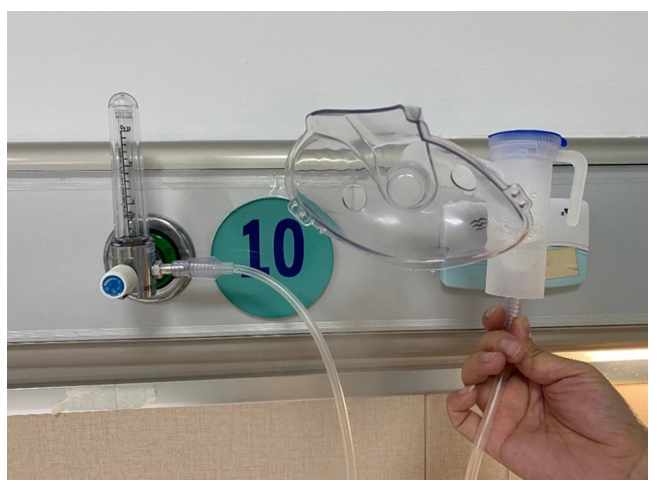

Figure 2 Assemble the nebulization device, oxygen flowmeter, and hospital wall oxygen supply device.

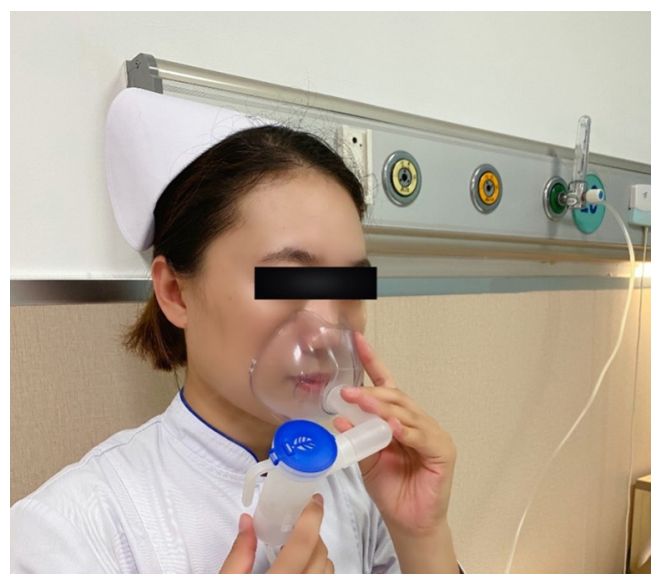

Figure 3 Demonstration of transnasal nebulization operation. This image is published with the participant's consent. methods are shown in Figures 2,3, respectively.

\section{Current development of transnasal nebulization in nasal disease}

Multiple studies have confirmed that short-term steroid transnasal nebulization is an effective and safe treatment option for patients with CRS. A randomized controlled trial involving patients after endoscopic sinus surgery for CRSwNP showed that the use of budesonide respules, particularly with a mucosal atomization device (MAD), was superior to fluticasone nasal spray (18). Recently, Zhang et al. (7) compared three approaches of corticosteroid delivery for the treatment of eosinophilic CRSwNP (ECRSwNP). They found that a short course of budesonide inhalation suspension via transnasal nebulization was more efficacious than budesonide nasal spray and safer than oral methylprednisolone regarding the HPA axis function. Another study compared the effect of three drug delivery modalities on olfactory function in CRS and found that sonic nebulization and oral administration were more effective than nasal spray (15). Transnasal nebulization could also affect the bleeding volume during endoscopic sinus surgery. Xing et al. reported that short-term preoperative use of budesonide inhalation suspension via transnasal nebulization could effectively reduce the inflammation of the nasal mucosa, eliminate mucosal edema, reduce the volume of nasal polyps, and significantly reduce intraoperative bleeding, especially for CRSwNP (19). Transnasal inhalation of nebulized budesonide suspension could be performed as a preoperative routine in patients with ECRSwNP because studies have shown that a short course of budesonide inhalation suspension via transnasal nebulization can quickly improve nasal symptoms and reduce the size of polyps without obvious HPA axis suppression $(20,21)$. Furthermore, short-term budesonide transnasal nebulization could achieve clinical improvement by regulating tissue remodeling, cytokine expression, and T cell subset distribution in ECRSwNP (12). Similarly, Thamboo et al. (16) suggested that short-term budesonide delivered via MAD for CRSsNP was a safe and effective method. Besides, Kanowitz et al. (17) found that topical budesonide via MAD might reduce the need for systemic prednisone for refractory postoperative CRS and improve overall assessment scores. Budesonide transnasal nebulization in CRS may achieve significant clinical improvements by regulating cytokine expression, $\mathrm{T}$ cell subsets, and tissue remodeling without serious side effects (22). 


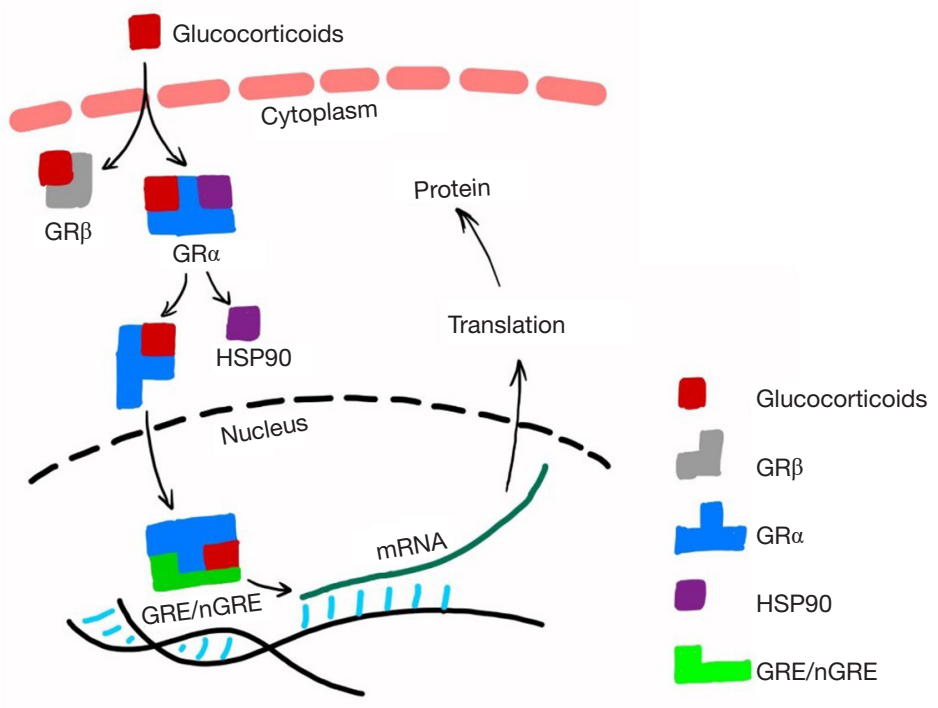

Figure 4 Mechanisms of glucocorticoid anti-inflammatory effects. GR is categorized into GR $\alpha$ and GR $\beta$ subtypes. The activation of GR $\alpha$ produces classical glucocorticoid effects, while GR $\beta$ acts as an antagonist against GR $\alpha$. When unactivated GR $\alpha$ combines with HSP90, the effect of GR $\alpha$ is hindered. When GR $\alpha$ binding to glucocorticoids with HSP90 dissociated, the activated glucocorticoid-receptor complex quickly translocates into the nucleus. Furthermore, it binds to the GRE, resulting in an increase in gene transcription, which in turn changes the level of mediator-related proteins. GR, glucocorticoid receptor; HSP90, heat shock protein 90; GRE, glucocorticoid response element; nGRE, negative glucocorticoid response element.

Transnasal nebulization has also been evaluated in other nasal diseases. Liu et al. (23) reported that nasal inhalation of budesonide, compared with oral inhalation, could control combined allergic rhinitis (AR) and asthma syndrome. Another study on perennial AR showed that compared to placebo, nebulized budesonide could improve symptoms and objective measures of nasal congestion, although it did not achieve statistical significance (24). The failure to reach statistical significance was considered to be related to the dose of active agents, the effect of the placebo, and the small number of participants. In addition, for patients with allergic fungal rhinosinusitis (AFRS) following endoscopic sinus surgery, in the budesonide transnasal nebulization group the recurrence rate was observed lower than that in the budesonide nasal sprays group, demonstrating the effectiveness of nebulized budesonide in treating patients with AFRS (25).

\section{Pharmacological mechanism of corticosteroids via transnasal nebulization}

\section{Anti-inflammation mechanisms of corticosteroids}

Glucocorticoids exert anti-inflammatory and immunosuppressive effects mainly through genomic and non-genomic pathways (26). The classic genomic effect is that glucocorticoids bind to glucocorticoid receptors (GRs) in the cytoplasm of target cells, thereby affecting the expression of genes involved in inflammation $(27,28)$ (Figure 4). The non-genomic effects include rapid onset and insensitivity to inhibitors of transcription and protein synthesis, which may be mainly related to the glucocorticoid membrane receptors, the direct effects on cell energy metabolism, and the signal pathways mediated by in vitro components of cytoplasmic receptors (29).

Nebulized inhalation therapy improves the absorption of glucocorticoids by nasal mucosa by allowing glucocorticoids to reach the deepest part of the nasal cavity, cover more mucosal surface area, and stay 3-4 times longer compared to nasal spray (7,30-33). In addition, nebulized inhalation can be useful in humidifying and cleaning the nasal mucosa.

\section{Metabolism of corticosteroids via transnasal nebulization}

After transnasal nebulization, about $30 \%$ of the drugs remain in the nasal mucosa, where they exert a pharmacological effect and are absorbed into the systemic 


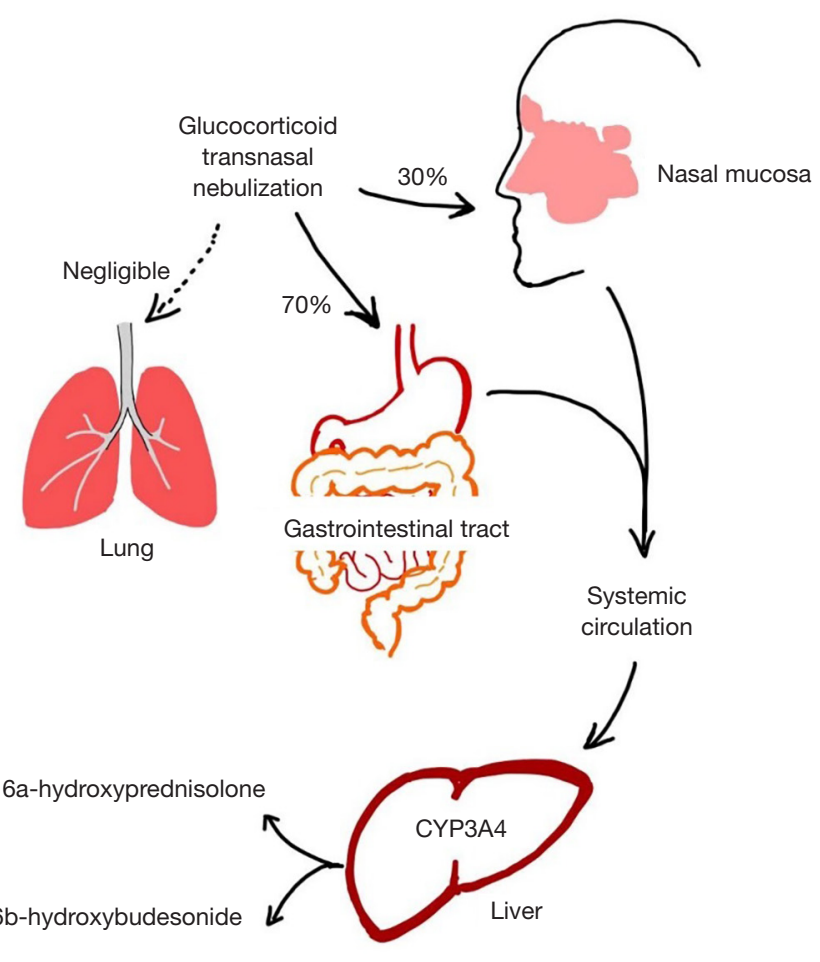

Figure 5 Metabolism of the glucocorticoid via transnasal nebulization.

circulation through the nasal venous system. Through the nasal mucociliary transport system, the remaining $70 \%$ of the drugs are transported to the nasopharynx and swallowed into the gastrointestinal tract, and then absorbed into the systemic circulation (21) (Figure 5).

Budesonide inhalation suspension (Pulmicort Respules, AstraZeneca) is a second-generation non-halogenated glucocorticoid, which has a strong affinity for GRs and is currently the only nebulized inhaled corticosteroid approved by U.S. Food and Drug Administration. Studies on patients with eosinophilic CRS have shown that budesonide transnasal nebulization significantly lowers the levels of interleukin (IL)-5 and eotaxin in nasal polyps and reduces eosinophil infiltration and T-helper type 2 (Th2) cell frequency $(7,12)$. In contrast, Th1/Th17-mixed inflammation, commonly associated with neutropenia, showed reduced sensitivity to glucocorticoids $(12,34)$. In addition, budesonide transnasal nebulization significantly increased the expression of transforming growth factor- $\beta$ and IL-10 in nasal polyps and the number of natural regulatory $\mathrm{T}$ cells and type 1 regulatory $\mathrm{T}$ cells (12). Budesonide transnasal nebulization could also significantly decrease the indicators of tissue remodeling, such as albumin, MMP-2, MMP-7, MMP-8, and MMP-9 and significantly increase collagen deposition and the levels of TIMP-1, TIMP-2, and TIMP-4 in nasal polyps $(7,12)$. The exact role of budesonide transnasal nebulization in CRS treatment remains to be investigated.

\section{Efficacy and safety of corticosteroids via transnasal nebulization}

\section{CRSwNP}

Corticosteroids are essential medications that target the pro-inflammatory pathway in CRSwNP. The antiinflammatory mechanisms of corticosteroid therapy include reduction in inflammatory cells, suppression of inflammatory cytokines and chemotactic factors, and regulation of tissue remodeling (22). Nebulize and spray are the two most common drug administration methods. Among them, nebulized droplets may target the sinuses more effectively than sprayed particles at sizes achieving optimal deposition for patients with CRS (35). Transnasal nebulization of corticosteroids is a new option for topical corticosteroid treatment of CRS.

A recent study (12) investigating the effect of 2 -week 
treatment with budesonide transnasal nebulization (1mg twice daily) in patients with ECRSwNP demonstrated significant inhibition of eosinophil infiltration, accompanied by lower eotaxin production. Concomitantly, Th2-biased inflammation was also significantly attenuated, as evidenced by a reduction in Th2 cell numbers and IL-5 levels in nasal polyps. Patients with CRSwNP achieved comparable improvement in symptom scores and reduction in polyp sizes after 2 -week budesonide transnasal nebulization and 4-week treatment with budesonide nasal spray (12). Similarly, a recent study demonstrated that the budesonide transnasal nebulization was safe and effective in delivering steroids to the sinuses of patients with CRS (16).

Importantly, steroid transnasal nebulization has comparable efficacy with oral steroids in CRSwNP management. A study comparing the efficacy and safety of steroids administered via the oral, intranasal spray, and transnasal nebulization routes in CRSwNP management suggested that a short course of steroid transnasal nebulization was more efficacious than steroid nasal spray and was safer than oral steroid regarding the effect on HPA axis function (7). Another study has shown that nebulized budesonide is equally effective in improving olfactory function in patients with CRS as oral prednisolone and significantly more effective than budesonide administered by nasal spray (15). A similar earlier study (17) also showed that topical nebulized budesonide effectively reduced the need for systemic prednisone and improved global assessment scores in refractory postoperative CRS patients. Topical nebulized steroid was also necessary for postoperative patients with CRS. Patients treated with budesonide after endoscopic sinus surgery for CRSwNP had greater improvement in 22-item Sinonasal Outcome Test (SNOT-22) and Lund-Kennedy scores compared to fluticasone at 6 months (18).

\section{CRSsNP}

Although intranasal corticosteroid treatment is the mainstay of treatment of patients with CRSsNP before and after endoscopic sinus surgery, few studies have investigated the application of corticosteroid nebulized inhalation therapy for CRSsNP. Only one study has focused on nebulized budesonide treatment for patients with CRSsNP (16), and several studies have investigated the effect of nebulized budesonide on mixed CRS population with CRSsNP and CRSwNP $(15,17,36)$. In a mixed CRS population, postoperative topical budesonide treatment via MAD reduced the need for systemic prednisone and improved both physician and patient global assessment scores in an average follow-up duration of 31.5 weeks (17). Oral or MAD treatment with corticosteroids has improved olfactory function and demonstrated better clinical effect than nasal spray (15).

Thamboo et al. (16) randomized 20 CRSsNP patients to take budesonide $(1 \mathrm{mg})$ via MAD or impregnated nasal saline irrigation (INSI) twice a day for 60 days. Although SNOT-22 score did not differ between the two treatment groups at 60 days, patients using MAD did show statistically significant improvement of SNOT-22 scores at 60 days compared to baseline (16). There was no indication of adrenal suppression in either treatment group based on the adrenocorticotropic hormone (ACTH) stimulation test. Nevertheless, the disconcerting trend towards the adrenal suppression threshold triggered the authors to conduct another investigation over a longer period of exposure to budesonide by MAD (36). Three of 100 patients with CRSsNP or CRSwNP demonstrated adrenal suppression as indicated by cosyntropin stimulation test after longterm budesonide MAD treatment with a mean treatment duration of 23.5 (range, 6-37) months; however, none of them had relevant symptomatology (36). In addition, $6 \%$ of the patients had elevated intraocular pressure (36).

In summary, short-term budesonide delivered via MAD seems safe and effective for CRSsNP patients. However, the efficacy of budesonide treatment via MAD should be confirmed in randomized controlled trials with larger sample sizes, and the side effects of long-term budesonide treatment via MAD warrants further investigations.

\section{$A R$}

Intranasal glucocorticoids have been included in clinical practice guidelines for the management of AR (37). Nevertheless, some patients with AR cannot benefit from nasal sprays because of the uneven distribution of the drug in the nasal mucosa. Evidence supports the use of intranasal nebulization for greater penetration of medicine due to the thicker deposition of aerosol particles compared to nasal sprays. A nebulization system that produces particles greater than $6.42 \mu \mathrm{m}$ in diameter can prolong aerosol retention in the nasal cavity (30).

In a randomized, double-blind, placebo-controlled study, children aged 8-14 years who suffered mild intermittent allergic asthma and AR were treated with nebulized beclomethasone dipropionate (nBDP, daily dose of 800 
$\mu \mathrm{g}$, twice per day) or placebo using a facial mask (38). After a 4-week treatment, nasal fractional exhaled nitric oxide was comparable between children treated with either nBDP or placebo; however, lower IL-5 level and higher $\mathrm{pH}$ in nasal exhaled breath condensate were detected in the $\mathrm{nBDP}$ group. In addition, the severity of the general status of asthma and rhinitis symptoms measured by the visual analogue scale significantly improved after treatment with nBDP. Another parallel, randomized, double-blind, placebo-controlled study enrolled AR adults who were treated by nebulized budesonide (daily dose of $250 \mu \mathrm{g}$, once per day) or placebo via a $\mathrm{NasoNeb}^{\circledR}$ device for 26 days (24). Compared to placebo, nebulized budesonide significantly improved the total nasal symptom scores, but no significant inter-group differences in nasal peak inspiratory flow, nasal volume measured by acoustic rhinometry, and rhinoconjunctivitis quality of life scores were detected. The existing evidence suggests that intranasal nebulization of glucocorticoids is an effective therapeutic strategy of AR. A better clinical efficacy may be expected at a higher dosage of glucocorticoids. Notably, these preliminary results are based on clinical pilot studies and require deeper exploration in the future.

Since partial agents can be absorbed into the blood circulation, nebulized inhalation of glucocorticoids may trigger adverse events; thus, long-term usage should be avoided. We conclude that nebulized glucocorticoids can be used for short-term treatment of asthma attacks and severe nasal symptoms in AR patients with or without asthma. High-quality clinical studies are required to validate the clinical significance of intranasal nebulization of glucocorticoids for AR treatment and standardize the indications, dosages, and courses of treatment regimens.

\section{Perioperative application}

Regarding the application of steroid transnasal nebulization in the perioperative period, administration of budesonide suspension, $2 \mathrm{~mL}$ twice a day for 1 week before surgery, can effectively reduce mucosal inflammation, the volume of nasal polyps, and intraoperative bleeding. The mechanism might be related to the reduction in local mucosal blood flow after inhalation of budesonide (19). Compared with oral corticosteroids, budesonide transnasal nebulization immediately before surgery can improve symptoms rapidly and has no obvious effect on adrenal cortex function, demonstrating its safety (21). In addition, compared with nasal spray, transnasal nebulization is more effective in improving postoperative symptoms (18). Some studies have also shown that for refractory sinusitis, continuous inhalation of budesonide suspension, $2 \mathrm{~mL}$ twice a day, can reduce the dosage of oral steroids (17). For refractory fungal sinusitis, $2 \mathrm{~mL}$ budesonide suspension inhaled twice a day and then gradually reduced to the maintenance dose once a week can reduce the recurrence rate (25).

\section{Long-term application/control after operation}

Because of the superior effect of corticosteroid nebulization over nasal spray for CRS treatment (22), it is reasonable to expect its long-term efficacy in treating patients with CRS after sinus surgery. However, studies in this field are scanty. Neubauer et al. (18) studied 32 patients with CRSwNP after endoscopic sinus surgery who were randomized to fluticasone nasal spray, budesonide respules via MAD, or budesonide respules via the vertex-to-floor position. At postoperative 6-month, patients on nasal budesonide nebulization therapy achieved the greatest reduction of SNOT-22 and Lund-Kennedy scores compared to those using fluticasone nasal spray and budesonide nasal drop.

Additionally, the safety of long-term corticosteroids nebulization is a matter of clinical importance. Recently, a cross-sectional study by Manji et al. (36) showed that longterm use (over 2 years) of budesonide nebulization via MAD in patients with recalcitrant CRS is associated with a risk of asymptomatic adrenal suppression detected on ACTH stimulation testing ( $3 \%$ of patients studied) and raised intraocular pressure based on applanation tonometry $(6 \%$ of patients studied). Although further investigations are necessary, this study suggests that long-term maintenance treatment with budesonide nebulization, particularly delivered via MAD, for patients with CRS should be used with caution and be monitored accordingly.

\section{Safety}

Safety of glucocorticoids is a concern for physicians due to potential systemic side effects, such as exacerbation of hypertension, weight gain, fluid retention, osteoporosis, and suppression of HPA axis, resulting from high-dose topical steroids (39-41).

The safety of short-term steroid transnasal nebulization during the CRS treatment is well documented $(7,12,16)$. The morning plasma cortisol levels of participants receiving budesonide inhalation for 2 weeks had no significant difference with those of placebo or baseline (12). 
Zhang et al. compared the effect of short-term oral methylprednisolone, budesonide transnasal nebulization, or budesonide nasal spray on ECRSwNP (7). The group receiving oral methylprednisolone showed significantly lower serum cortisol levels than the other two groups, while no difference in serum cortisol levels between the latter two groups was observed, and no adverse events were reported. Another study assessed the safety of budesonide inhalation for 60 days (16). There was no detectable plasma budesonide, serious adverse events, or sign of adrenal suppression in MAD group and nasal irrigation group. In conclusion, the administration of corticosteroids via transnasal nebulization limits systemic exposure compared with oral corticosteroids and reduces the risk of systemic side effects. The risk of short-term steroid transnasal nebulization steroids is similar to that of steroid nasal spray or irrigation prescribed in CRS (42-44).

\section{Special application}

\section{Nebulization therapy for the nasal diseases in special population}

Intranasal steroid therapy has proved to be the most effective treatment for nasal diseases, including AR and various forms of CRS. However, the persistence of efficacy using topical intranasal steroid sprays should be improved (45). Numerous clinical studies have reported the efficacy and safety of nasal nebulization inhalation of steroids $(7,12,20-22)$. Compared to the nasal spray, nebulization therapy system has minimal drug loss and is significantly more effective $(15,39)$. Further, nebulized droplets may target the sinuses more effectively than sprayed particles at sizes achieving optimal deposition (35).

The safety of corticosteroids is a common concern in special populations, such as pregnant women, children, and older people, due to potential systemic side effects. Intranasal corticosteroid therapy is considered safe for the pediatric population, but there is little evidence on the use of steroid transnasal nebulization in children. A multicenter, randomized, double-blind, placebo-controlled, parallelgroup 76-week safety study evaluated the effect of intranasal corticosteroids on growth velocity in children. A slight reduction in growth velocity after 52 weeks of treatment using Fluticasone furoate nasal spray (FFNS) $110 \mathrm{mcg}$ once daily was observed, with no other safety issue identified. The authors suggested that the starting dosage of intranasal corticosteroids in children should be FFNS $55 \mathrm{mcg}$ once daily (46). Meanwhile, another study demonstrated that treatment with mometasone furoate nasal spray $100 \mathrm{mcg}$ once daily for 1 year was well tolerated in children aged 6-11 years (47). Although aerosol therapy with nasal cannula is a common treatment in children, a new nasal nebulized system should be specifically designed for this pediatric population (48). According to current guidelines, intranasal steroids are considered safe during pregnancy. Some authors suggest fluticasone during pregnancy due to the absence of adverse outcomes $(49,50)$. However, budesonide is the only category B intranasal steroid of U.S. Food and Drug Administration pregnancy risk categories for pregnant women (51).

The clinical trials on the safety of intranasal steroids in older people are few. Some randomized controlled studies evaluated the safety of mometasone furoate and fluticasone in patients over 65 years old (52). The most frequent side effects in these patients include mouth and nose dryness, burning of the nasal mucosa, nasal crusting, and epistaxis. However, there is no evidence that intranasal steroids can change bone metabolism or cause ocular adverse effects $(53,54)$. In a nutshell, intranasal steroid therapy should be considered a sufficiently safe treatment option for children, pregnant women, and the elderly. More studies are needed to evaluate the efficacy and safety of the steroids nebulization delivery system in these special populations.

\section{Application of nebulized inbalation therapy during the coronavirus disease (COVID-19) pandemic}

The current COVID-19 pandemic raises critical questions on whether pre-morbid use or continued use of nebulized inhaled corticosteroids affects the outcomes of acute respiratory infection caused by the coronavirus. Because of immunosuppressive effects, many physicians are concerned about the inhalation of corticosteroids by COVID-19 positive patients.

Currently, there is no evidence on whether the use of nebulized inhaled corticosteroids modifies the susceptibility to or severity of COVID-19. Previous studies have shown that inhaled corticosteroids, especially high doses, may raise the risk of upper respiratory tract infections in adult patients with asthma and chronic obstructive pulmonary disease $(55,56)$. However, regular use of inhaled corticosteroids may not increase the risk of upper respiratory tract infections in children with asthma (57). Recent studies have reported lower expression of angiotensin-converting enzyme 2 (ACE2) and transmembrane protease serine 
2 (TMPRSS2) in sputum cells of patients with asthma who are on inhaled corticosteroid therapy, indicating that inhaled corticosteroids might alleviate the susceptibility to COVID-19 infection or COVID-19 morbidity $(58,59)$. In contrast, Wang et al. showed that ACE2 expression was significantly increased in nasal tissues of patients with nonECRSwNP compared to those of patients with ECRSwNP and control subjects, while TMPRSS2 expression was decreased in nasal tissues of patients with CRSwNP compared to control subjects (60). Moreover, glucocorticoid treatment reduced ACE2 expression in nasal tissues of nonECRSwNP patients, but not of ECRSwNP patients, while TMPRSS2 expression was not affected (60). Recently, it has been shown that the ongoing use of inhaled corticosteroids does not increase the risk of hospitalization of COVID-19 patients with asthma (61-64). Nevertheless, further research is needed to assess whether nebulized inhaled corticosteroids have a protective effect in patients with COVID-19, particularly those with AR and rhinosinusitis.

Notably, since stopping corticosteroid therapy leads to the potentially dangerous worsening of the baseline airway diseases, patients with AR, CRS, and asthma should not discontinue their prescribed inhaled corticosteroid treatment during the COVID-19 pandemic (65-67).

\section{Points for attention in nebulization therapy}

\section{Preparation before nebulization}

Compared to systemic and topical steroid administration, transnasal steroid nebulization is an effective and safe treatment option, with a lower incidence of serious side effects in patients with CRS $(20,21)$. Inhaled particles are easily distributed in the nasal cavity, larynx, and pharynx; however, several local side effects, such as infection, hoarseness, sore throat, and airway hypersensitivity, should also be emphasized $(68,69)$. Before nebulization, the following items should be prepared well $(7,12,68)$ : (I) the breathing tube and intranasal plug in the pulsating atomization device should be disposable to avoid crosscontamination. (II) Before aerosol inhalation, patients should be adjusted to a comfortable position for efficient distribution in the nasal cavity and paranasal sinus, thus achieving the best usage of the nebulized medicine. (III) Staff in charge of the device should adhere to hand hygiene procedures to reduce pathogenic bacteria dissemination among patients. (IV) Caregivers should be wary of complications, such as aerosol-induced airway spasm and irritable cough, during the therapeutic process. (V) According to pharmacological guidelines, single dosedependent inhaled steroid suspension is recommended instead of combined usage with other drugs.

\section{Procedures of nebulization}

(I) Studies have shown that the fine particles with an aerodynamic diameter of $10-14 \mu \mathrm{m}$ are suitable for absorption in nasal mucosa as they are more likely to be deposited in paranasal sinuses and ostiomeatal complex (35). Clinically, one of the most common inhaled corticosteroids is budesonide suspension, with a once-daily dose of $1 \mathrm{mg} / 2 \mathrm{~mL}$ and nebulization time of $20-30 \mathrm{~min}(7,12,20,21)$. The concentration should be appropriate for steroid nebulization, with inhaled speed increasing gradually instead of suddenly $(68,69)$. (II) Oronasal care, including removal of the secretion and food residue from mouth and nose cleaning before aerosol inhalation, should be done to decrease the risk of local fungal infection and aspiration (70). (III) In case of nasal dryness and nasal bleeding during nebulization, nasal irrigation with sodium chloride can be performed. Other options include endoscopic examination and management (70). (IV) For patients with severe nasal septum deviation, surgical intervention should be considered before nebulization (35). (V) Additional focus is needed for systemic complications during nebulization in patients with cardiac and renal insufficiency and autoimmune dysfunction or in the elderly and weak individuals $(7,12)$.

\section{Conclusions}

Compared to nasal spray, transnasal nebulized inhalation ensures broad exposure of the steroids to the nasal cavity and sinus mucosa, prolonging steroid retention. The effect of transnasal nebulized inhalation of corticosteroids is better than those of nasal spray. Transnasal corticosteroid nebulization can be used for patients with CRSsNP, CRSwNP (especially eCRSwNP), and AR. Up to date, the recommended course of budesonide transnasal nebulization treatment is $1 \mathrm{mg}$ twice daily for 2 weeks in ECRSwNP. The environmental and equipment requirements for inhalation of corticosteroids via nasal nebulization are easily met. Therefore, performing transnasal nebulized inhalation for patients with CRS in the clinic is convenient.

Further, more prospective research is needed to provide more solid evidence to standardize the use of transnasal nebulized inhalation and optimize treatment regimens. 
Accumulation of additional evidence may benefit more patients with CRS in the future.

\section{Acknowledgments}

Funding: This work was supported by grants from the national natural science foundation of China (81630023, 81970850, 81870698, and 82025010), National Key R \& D Program of China (2018YFC0116800), CAMS Innovation Fund for Medical Sciences (CIFMS) (2019-I2M-5-022, 2021-I2M-C\&T-B-098), the Program for Changjiang Scholars and Innovative Research Team (IRT13082), and Beijing Municipal Science and Technology Project (Z181100001618002).

\section{Footnote}

Reporting Checklist: The authors have completed the RIGHT reporting checklist. Available at https://dx.doi. org/10.21037/jtd-21-1142

Conflicts of Interest: All authors have completed the ICMJE uniform disclosure form (available at https://dx.doi. org/10.21037/jtd-21-1142). The authors have no other conflicts of interest to declare.

Ethical Statement: The authors are accountable for all aspects of the work in ensuring that questions related to the accuracy or integrity of any part of the work are appropriately investigated and resolved.

Open Access Statement: This is an Open Access article distributed in accordance with the Creative Commons Attribution-NonCommercial-NoDerivs 4.0 International License (CC BY-NC-ND 4.0), which permits the noncommercial replication and distribution of the article with the strict proviso that no changes or edits are made and the original work is properly cited (including links to both the formal publication through the relevant DOI and the license). See: https://creativecommons.org/licenses/by-nc-nd/4.0/.

\section{References}

1. Fokkens WJ, Lund VJ, Hopkins C, et al. European Position Paper on Rhinosinusitis and Nasal Polyps 2020. Rhinology 2020;58:1-464.

2. Shi JB, Fu QL, Zhang H, et al. Epidemiology of chronic rhinosinusitis: results from a cross-sectional survey in seven Chinese cities. Allergy 2015;70:533-9.

3. Hopkins C. Chronic Rhinosinusitis with Nasal Polyps. N Engl J Med 2019;381:55-63.

4. Levy JM, Mace JC, DeConde AS, et al. Improvements in psychological dysfunction after endoscopic sinus surgery for patients with chronic rhinosinusitis. Int Forum Allergy Rhinol 2016;6:906-13.

5. Lou H, Wang C, Zhang L. Endotype-driven precision medicine in chronic rhinosinusitis. Expert Rev Clin Immunol 2019;15:1171-83.

6. Fokkens WJ, Lund VJ, Mullol J, et al. EPOS 2012: European position paper on rhinosinusitis and nasal polyps 2012. A summary for otorhinolaryngologists. Rhinology 2012;50:1-12.

7. Zhang $\mathrm{Y}$, Lou $\mathrm{H}$, Wang $\mathrm{Y}$, et al. Comparison of Corticosteroids by 3 Approaches to the Treatment of Chronic Rhinosinusitis With Nasal Polyps. Allergy Asthma Immunol Res 2019;11:482-97.

8. Gelardi M, Passalacqua G, Fiorella ML, et al. Assessment of biofilm by nasal cytology in different forms of rhinitis and its functional correlations. Eur Ann Allergy Clin Immunol 2013;45:25-9.

9. Macchi A, Terranova P, Digilio E, et al. Hyaluronan plus saline nasal washes in the treatment of rhino-sinusal symptoms in patients undergoing functional endoscopic sinus surgery for rhino-sinusal remodeling. Int $\mathrm{J}$ Immunopathol Pharmacol 2013;26:137-45.

10. Cantone E, Castagna G, Sicignano S, et al. Impact of intranasal sodium hyaluronate on the short-term quality of life of patients undergoing functional endoscopic sinus surgery for chronic rhinosinusitis. Int Forum Allergy Rhinol 2014;4:484-7.

11. Casale M, Sabatino L, Frari V, et al. The potential role of hyaluronan in minimizing symptoms and preventing exacerbations of chronic rhinosinusitis. Am J Rhinol Allergy 2014;28:345-8.

12. Wang C, Lou H, Wang X, et al. Effect of budesonide transnasal nebulization in patients with eosinophilic chronic rhinosinusitis with nasal polyps. J Allergy Clin Immunol 2015;135:922-9.e6.

13. Stein SW, Thiel CG. The History of Therapeutic Aerosols: A Chronological Review. J Aerosol Med Pulm Drug Deliv 2017;30:20-41.

14. Labiris NR, Dolovich MB. Pulmonary drug delivery. Part II: the role of inhalant delivery devices and drug formulations in therapeutic effectiveness of aerosolized medications. Br J Clin Pharmacol 2003;56:600-12.

15. Reychler G, Colbrant C, Huart C, et al. Effect of three- 
drug delivery modalities on olfactory function in chronic sinusitis. Laryngoscope 2015;125:549-55.

16. Thamboo A, Manji J, Szeitz A, et al. The safety and efficacy of short-term budesonide delivered via mucosal atomization device for chronic rhinosinusitis without nasal polyposis. Int Forum Allergy Rhinol 2014;4:397-402.

17. Kanowitz SJ, Batra PS, Citardi MJ. Topical budesonide via mucosal atomization device in refractory postoperative chronic rhinosinusitis. Otolaryngol Head Neck Surg 2008;139:131-6.

18. Neubauer PD, Schwam ZG, Manes RP. Comparison of intranasal fluticasone spray, budesonide atomizer, and budesonide respules in patients with chronic rhinosinusitis with polyposis after endoscopic sinus surgery. Int Forum Allergy Rhinol 2016;6:233-7.

19. Xing J, Liu X, Tian Y. The effect of budesonide inhalation suspension via transnasal nebulization to the volume of bleeding during endoscopic sinus surgery. Lin Chung Er Bi Yan Hou Tou Jing Wai Ke Za Zhi 2014;28:1077-8.

20. Wang C, Lou H, Lou W, et al. The efficacy and safety of a short course of budesonide inhalation suspension via transnasal nebulization in chronic rhinosinusitis with nasal polyps. Lin Chung Er Bi Yan Hou Tou Jing Wai Ke Za Zhi 2012;26:347-50.

21. Lou H, Wang C, Zhang L. The efficacy and safety of budesonide inhalation suspension via transnasal nebulization compared with oral corticosteroids in chronic rhinosinusitis with nasal polyps. Lin Chung Er Bi Yan Hou Tou Jing Wai Ke Za Zhi 2015;29:792-6.

22. Lou H, Wang C, Zhang L. Steroid transnasal nebulization in the treatment of chronic rhinosinusitis. Curr Opin Allergy Clin Immunol 2016;16:39-44.

23. Liu Y, Fu Q, Zhang Y, et al. Treatment of combined allergic rhinitis and asthma syndrome by nasal inhalation of budesonide. Chinese Journal of Asthma (Electronic Version) 2009;3:113-5.

24. Brown K, Lane J, Silva MP, et al. A pilot study of the effects of intranasal budesonide delivered by NasoNeb® on patients with perennial allergic rhinitis. Int Forum Allergy Rhinol 2014;4:43-8.

25. Dai Q, Duan C, Liu Q, et al. Effect of nebulized budesonide on decreasing the recurrence of allergic fungal rhinosinusitis. Am J Otolaryngol 2017;38:321-4.

26. Zen M, Canova M, Campana C, et al. The kaleidoscope of glucorticoid effects on immune system. Autoimmun Rev 2011;10:305-10.

27. Lemanske RF Jr, Busse WW. 6. Asthma. J Allergy Clin Immunol 2003;111:S502-19.
28. Grbesa I, Hakim O. Genomic effects of glucocorticoids. Protoplasma 2017;254:1175-85.

29. Panettieri RA, Schaafsma D, Amrani Y, et al. Nongenomic Effects of Glucocorticoids: An Updated View. Trends Pharmacol Sci 2019;40:38-49.

30. Frank DO, Kimbell JS, Pawar S, et al. Effects of anatomy and particle size on nasal sprays and nebulizers. Otolaryngol Head Neck Surg 2012;146:313-9.

31. Lee J, Kim Y, Park C, et al. Comparison between dexmedetomidine and remifentanil for controlled hypotension and recovery in endoscopic sinus surgery. Ann Otol Rhinol Laryngol 2013;122:421-6.

32. Möller W, Schuschnig U, Khadem Saba G, et al. Pulsating aerosols for drug delivery to the sinuses in healthy volunteers. Otolaryngol Head Neck Surg 2010;142:382-8.

33. DeYoung K, Wentzel JL, Schlosser RJ, et al. Systematic review of immunotherapy for chronic rhinosinusitis. Am J Rhinol Allergy 2014;28:145-50.

34. Wen $W$, Liu $W$, Zhang L, et al. Increased neutrophilia in nasal polyps reduces the response to oral corticosteroid therapy. J Allergy Clin Immunol 2012;129:1522-8.e5.

35. Farzal Z, Basu S, Burke A, et al. Comparative study of simulated nebulized and spray particle deposition in chronic rhinosinusitis patients. Int Forum Allergy Rhinol 2019;9:746-58.

36. Manji J, Singh G, Okpaleke C, et al. Safety of longterm intranasal budesonide delivered via the mucosal atomization device for chronic rhinosinusitis. Int Forum Allergy Rhinol 2017;7:488-93.

37. Cheng L, Chen J, Fu Q, et al. Chinese Society of Allergy Guidelines for Diagnosis and Treatment of Allergic Rhinitis. Allergy Asthma Immunol Res 2018;10:300-53.

38. Profita M, Riccobono L, Bonanno A, et al. Effect of nebulized beclomethasone on airway inflammation and clinical status of children with allergic asthma and rhinitis: a randomized, double-blind, placebo-controlled study. Int Arch Allergy Immunol 2013;161:53-64.

39. Allen DB. Systemic effects of intranasal steroids: an endocrinologist's perspective. J Allergy Clin Immunol 2000;106:S179-90.

40. Boner AL. Effects of intranasal corticosteroids on the hypothalamic-pituitary-adrenal axis in children. J Allergy Clin Immunol 2001;108:S32-9.

41. Priftis KN, Papadimitriou A, Gatsopoulou E, et al. The effect of inhaled budesonide on adrenal and growth suppression in asthmatic children. Eur Respir J 2006;27:316-20.

42. Bhalla RK, Payton K, Wright ED. Safety of budesonide 
in saline sinonasal irrigations in the management of chronic rhinosinusitis with polyposis: lack of significant adrenal suppression. J Otolaryngol Head Neck Surg 2008;37:821-5.

43. Sachanandani NS, Piccirillo JF, Kramper MA, et al. The effect of nasally administered budesonide respules on adrenal cortex function in patients with chronic rhinosinusitis. Arch Otolaryngol Head Neck Surg 2009;135:303-7.

44. Welch KC, Thaler ER, Doghramji LL, et al. The effects of serum and urinary cortisol levels of topical intranasal irrigations with budesonide added to saline in patients with recurrent polyposis after endoscopic sinus surgery. Am J Rhinol Allergy 2010;24:26-8.

45. Lund VJ, Black JH, Szabó LZ, et al. Efficacy and tolerability of budesonide aqueous nasal spray in chronic rhinosinusitis patients. Rhinology 2004;42:57-62.

46. Lee LA, Sterling R, Máspero J, et al. Growth velocity reduced with once-daily fluticasone furoate nasal spray in prepubescent children with perennial allergic rhinitis. J Allergy Clin Immunol Pract 2014;2:421-7.

47. Ratner PH, Meltzer EO, Teper A. Mometasone furoate nasal spray is safe and effective for 1-year treatment of children with perennial allergic rhinitis. Int J Pediatr Otorhinolaryngol 2009;73:651-7.

48. Kesavan S, Amirav I. Is aerosol delivery by high-flow nasal cannula in children an effective alternative to face mask aerosol nebulization? Pediatr Pulmonol 2019;54:1873-4.

49. Ellegård EK, Hellgren M, Karlsson NG. Fluticasone propionate aqueous nasal spray in pregnancy rhinitis. Clin Otolaryngol Allied Sci 2001;26:394-400.

50. Choi JS, Han JY, Kim MY, et al. Pregnancy outcomes in women using inhaled fluticasone during pregnancy: a case series. Allergol Immunopathol (Madr) 2007;35:239-42.

51. Gonzalez-Estrada A, Geraci SA. Allergy Medications During Pregnancy. Am J Med Sci 2016;352:326-31.

52. Ridolo E, Caminati M, Martignago I, et al. Allergic rhinitis: pharmacotherapy in pregnancy and old age. Expert Rev Clin Pharmacol 2016;9:1081-9.

53. Wilson AM, Sims EJ, McFarlane LC, et al. Effects of intranasal corticosteroids on adrenal, bone, and blood markers of systemic activity in allergic rhinitis. J Allergy Clin Immunol 1998;102:598-604.

54. Garbe E, Suissa S. Inhaled corticosteroids and the risk of cataracts. N Engl J Med 1997;337:1555.

55. Yang $M$, Zhang $\mathrm{Y}$, Chen $\mathrm{H}$, et al. Inhaled corticosteroids and risk of upper respiratory tract infection in patients with asthma: a meta-analysis. Infection 2019;47:377-85.
56. Yang M, Chen H, Zhang Y, et al. Long-term use of inhaled corticosteroids and risk of upper respiratory tract infection in chronic obstructive pulmonary disease: a meta-analysis. Inhal Toxicol 2017;29:219-26.

57. Cazeiro C, Silva C, Mayer S, et al. Inhaled Corticosteroids and Respiratory Infections in Children With Asthma: A Meta-analysis. Pediatrics 2017;139:e20163271.

58. Peters MC, Sajuthi S, Deford P, et al. COVID-19related Genes in Sputum Cells in Asthma. Relationship to Demographic Features and Corticosteroids. Am J Respir Crit Care Med 2020;202:83-90.

59. Liu S, Zhi Y, Ying S. COVID-19 and Asthma: Reflection During the Pandemic. Clin Rev Allergy Immunol 2020;59:78-88.

60. Wang M, Bu X, Fang G, et al. Distinct expression of SARS-CoV-2 receptor ACE2 correlates with endotypes of chronic rhinosinusitis with nasal polyps. Allergy 2021;76:789-803.

61. Chhiba KD, Patel GB, Vu THT, et al. Prevalence and characterization of asthma in hospitalized and nonhospitalized patients with COVID-19. J Allergy Clin Immunol 2020;146:307-14.e4.

62. Liao SY, Petrache I, Fingerlin TE, et al. Association of inhaled and systemic corticosteroid use with Coronavirus Disease 2019 (COVID-19) test positivity in patients with chronic pulmonary diseases. Respir Med 2021;176:106275.

63. Bloom CI, Drake TM, Docherty AB, et al. Risk of adverse outcomes in patients with underlying respiratory conditions admitted to hospital with COVID-19: a national, multicentre prospective cohort study using the ISARIC WHO Clinical Characterisation Protocol UK. Lancet Respir Med 2021;9:699-711.

64. Choi JC, Jung SY, Yoon UA, et al. Inhaled Corticosteroids and COVID-19 Risk and Mortality: A Nationwide Cohort Study. J Clin Med 2020;9:3406.

65. Yao Y, Wang H, Liu Z. Expression of ACE2 in airways: Implication for COVID-19 risk and disease management in patients with chronic inflammatory respiratory diseases. Clin Exp Allergy 2020;50:1313-24.

66. Klimek L, Jutel M, Bousquet J, et al. Management of patients with chronic rhinosinusitis during the COVID-19 pandemic-An EAACI position paper. Allergy 2021;76:677-88.

67. Bousquet J, Akdis CA, Jutel M, et al. Intranasal corticosteroids in allergic rhinitis in COVID-19 infected patients: An ARIA-EAACI statement. Allergy 2020;75:2440-4.

68. Xu W, Dong P, Gu Q, et al. Expert consensus on the 
application of aerosol inhalation therapy of pharyngeal diseases. Chinese Archives of Otolaryngology-Head and Neck Surgery 2019;26:231-8.

69. Wu J, Lu AD, Zhang LP, et al. Study of clinical outcome and prognosis in pediatric core binding factor-acute myeloid leukemia. Zhonghua Xue Ye Xue Za Zhi

Cite this article as: Wang C, Cheng L, Li H, Liu Z, Lou H, Shi J, Sun Y, Wang D, Yang Q, Yu H, Zhao C, Zhu D, Cheng F, Li Y, Liao B, Lu M, Meng C, Shen S, Sun Y, Zheng R, Zhang L; on behalf of the Group of Otorhinolaryngology and Ophthalmology, Chinese Society of Allergy (CSA). Chinese expert recommendation on transnasal corticosteroid nebulization for the treatment of chronic rhinosinusitis 2021. J Thorac Dis 2021;13(11):6217-6229. doi: 10.21037/jtd-21-1142
2019;40:52-7.

70. Compiling Group of "Expert Consensus on Rational Use of Nebulized Inhalation Therapy" CPBoCMA. Expert consensus on the rational use of nebulized inhalation therapy (2019 edition). Herald of Medicine 2019;38:135-46. 